\title{
La coulée continue des aciers. Un exemple de développement technique où l'étroite coopération entre métallurgistes, constructeurs et exploitants ont été d'une grande fécondité
}

\author{
Jean Saleil ${ }^{a, *}$ and Jean Le Coze ${ }^{b}$ \\ Cercle d'Études des Métaux, École Nationale Supérieure des Mines, 42023 Saint-Étienne Cedex 2, France
}

Reçu le 9 février 2018 / Accepté le 17 septembre 2018

\begin{abstract}
Résumé. Cet article est composé de quatre parties complémentaires l'une de l'autre mais publiées séparément. Dans la partie I, se trouve une description du fonctionnement des machines de coulée continue et l'historique du remarquable développement de cette technique de coulée appliquée aux aciers. La partie II présente les multiples problèmes qui furent résolus pour arriver à faire correctement fonctionner ces machines et leur permettre de délivrer des produits de qualité. Dans la partie III, sont développées les questions métallurgiques posées par la solidification des produits en continu et la complexité inhérente à chaque famille d'acier (bas carbone, inoxydables, alliés). La partie IV décrit quelques types particuliers de machines et notamment celles destinées à la production de bandes, avec l'ensemble des problèmes spécifiques posés par ces techniques.
\end{abstract}

Mots clés : historique du procédé / problèmes techniques de fonctionnement / problèmes métallurgiques / qualité des produits / aciers bas carbone / inoxydables / alliés / coulées de brames minces / coulée de bandes

\begin{abstract}
Continuous casting of steels. Part I: major steps of steels conti-casting development. This survey of continuous casting in steelmaking is divided in four parts. The first one contains the history of the development of the process technology. The second one is a description of the numerous problems, which were to be solved in order that the casting machines should be able to deliver high quality products. The third part deals with metallurgy and solidification problems, taking into account the great diversity of solidification patterns of various steel grades (low carbon steels, stainless steels, high carbon steels...). The fourth part describes some specific machines with the problems related to their operation, and particularly the technology of strip casting.
\end{abstract}

Keywords: process history / technical problems / metallurgical problems / products quality / low carbon steels / stainless steels / alloyed steels / thin slab casting / strip casting

En hommage au Dr Manfred Wolf (1937-2001) spécialiste de réputation mondiale de la coulée continue, aux équipes de chercheurs de l'IRSID et des laboratoires universitaires qui ont apporté une contribution significative à la mâ̂trise métallurgique du procédé, aux constructeurs de machines de coulée qui ont su rechercher avec obstination, trouver et appliquer industriellement, les solutions technologiques aux problèmes posés et aux exploitants aciéristes qui ont eu la volonté et

\footnotetext{
* e-mail: cemetaux@emse.fr

a Ingénieur civil des mines (Nancy 59), carrière dans les sociétés Ugine (Moutiers), Imphy, Creusot-Loire, Ascometal ; longtemps en charge des développements en matière de procédés d'élaboration.

b Ingénieur civil des mines (Saint-Étienne 63), Dr. Es Sciences, ex-enseignant chercheur ENSMSE.
}

la persévérance de valider en production les technologies proposées.

«L'objet d'un art est l'usage des moyens disponibles en vue d'une fin que l'on se propose. » Clausewitz (17801831).

Cette citation, relative à la stratégie militaire, qui évoque la combinaison des moyens disponibles ordonnés à une fin précise, s'applique tout particulièrement aux efforts intenses qui ont accompagné au fil des décennies le développement de la coulée continue en sidérurgie, dans la mesure où les ingénieurs ont mobilisé massivement leur art, au sens que prend le terme dans l'expression «Arts et Métiers», pour résoudre les problèmes technologiques et métallurgiques que posait ce mode de coulée de l'acier, et où les dirigeants ont placé ces développements au cœur des stratégies des entreprises sidérurgiques. 
La contribution de Jacques Barbé au présent article a été essentielle: par la mise à disposition de son abondante documentation et par ses nombreuses remarques et rectifications. Par sa carrière d'ingénieur dans la société d'engineering sidérurgique CLECIM, J. Barbé est une des rares personnes en France qui a participé, presque de bout en bout, à l'essor du procédé de coulée continue, depuis les premières machines installées en France, jusqu'au développement du projet Myosotis de la coulée de bandes. En avril 1992, au Congrès de Toronto (75th steel making conference), le Dr Wolf publiait un premier bilan historique de la coulée continue et son article se concluait par les photos de treize personnalités internationales qui avaient contribué de manière décisive aux progrès de la coulée continue des aciers. Déjà J. Barbé y figurait aux côtés de S. Junghans et I. Rossi.

\section{Introduction}

La coulée continue de l'acier, un vieux rêve des sidérurgistes poursuivi depuis qu'ils ont appris à la fin du $19^{\mathrm{e}}$ siècle à élaborer le métal par une voie en phase totalement liquide, s'est imposée progressivement au cours de la seconde moitié du $20^{\mathrm{e}}$ siècle dans tous les secteurs de l'activité sidérurgique: produits longs, produits plats, aciers courants, aciers spéciaux de construction, aciers inoxydables, au point que la coulée discontinue en lingots ne subsiste plus guère que pour des besoins très spécifiques : ébauches pour refusion, produits bruts de coulée destinés à la grosse forge ou aux tôles de très forte épaisseur. Pour parvenir à cette généralisation du procédé, les ingénieurs ont dû surmonter un grand nombre de problèmes techniques et métallurgiques, rendus encore plus complexes par leur imbrication. Leur élucidation a bien souvent fait appel aux meilleures équipes de chercheurs en métallurgie des centres de $R \& D$ de par le monde et leur résolution doit beaucoup à l'imagination créatrice et à l'ingéniosité des technologues. La constitution minutieuse d'une vaste défectologie et l'analyse très précise des causes métallurgiques et techniques de ces défauts ont joué un grand rôle dans la maîtrise progressive de la coulée continue et dans son extension à l'ensemble du catalogue des aciers.

Nous avons assisté, essentiellement au cours de la seconde moitié du $20^{\mathrm{e}}$ siècle, à un développement technologique majeur, associant R\&D et industrialisation, actions qui mobilisèrent des compétences aussi diverses que celles des métallurgistes, constructeurs/mécaniciens, aciéristes exploitants, réfractoristes, automaticiens. Ces efforts ont été rendus possibles par une intense activité de formation à la métallurgie des acteurs du terrain à tous les niveaux de compétences, avec le concours des organismes de formation académiques et professionnels. On peut mesurer le foisonnement technologique autour de la coulée continue au nombre considérable de brevets déposés sur le sujet. C'est ce couplage fécond de la réflexion métallurgique et de l'inventivité technologique, soutenu d'abord par le dynamisme et la volonté des derniers maîtres de forge, puis par des dirigeants visionnaires, sous la pression des exigences qualitatives et de productivité, que nous nous proposons d'exposer dans les diverses parties du présent article. Cette pression économique stimulante se manifesta à la fois par le développement de nouveaux marchés, l'apparition de nouvelles exigences qualitatives, l'apparition de nouveaux acteurs sidérurgiques. La conséquence fut qu'en cinquante ans le paysage sidérurgique mondial fut radicalement changé et dans ses techniques et dans ses acteurs.

Bien entendu cette apparition de la coulée continue n'était pas sans conséquences sur la conception des usines sidérurgiques puisqu'elle visait à supprimer une étape jusqu'alors incontournable de la mise en forme à chaud tant qu'on restait en voie lingots : celui du dégrossissage de ces lingots par un train spécifique de laminoir à chaud: le blooming (produits longs) ou le slabbing (produits plats). À partir des années 1960, pour chaque conception d'usine nouvelle ou pour toute modernisation d'une aciérie existante, se posait donc le dilemme du maintien de la voie lingots ou du passage en coulée continue. La réponse apportée dépendait en grande partie du carnet de nuances élaborées par l'aciérie considérée, car les technologies disponibles en coulée continue ne permettaient pas toujours de garantir les qualités requises par les nuances les plus pointues du carnet de commande, même si la diversité croissante des options technologiques disponibles permettait d'adapter de mieux en mieux la coulée continue aux cas particuliers. Le dilemme du passage ou non en CC, était d'autant plus crucial que l'option coulée continue était pratiquement irréversible puisqu'elle impliquait le plus souvent l'arrêt du train dégrossisseur des lingots. Soulignons une nouvelle fois que sans de grands industriels visionnaires qui s'acharnèrent à soutenir le développement du procédé malgré les difficultés technologiques et qualitatives considérables qu'il présentait à ses débuts, et qui assumèrent les risques d'introduction de ces équipements nouveaux dans les filières industrielles, la coulée continue n'aurait pas connu l'essor que nous constatons. Dès que les technologies de base furent assurées, le développement de la coulée continue fut rapide pour les nuances les plus courantes (par exemple, le rond à béton). Il le fut beaucoup moins pour certains aciers spéciaux puisqu'on observe, lors de l'actuelle décennie, la levée des derniers obstacles à la coulée continue pour des nuances qui longtemps l'exclurent, telles les nuances d'aciers pour roulements. Les produits plats occupèrent une place considérable dans ce développement d'abord pour les aciers au carbone, puis pour les aciers inoxydables: la pression des exigences en matière de qualité de surface, particulièrement forte pour les produits plats minces, fut à l'origine des principaux progrès notamment autour du fonctionnement de la tête de machine, du contrôle électromagnétique de la solidification, et du supportage des lignes.

Dans les 40 années du développement intensif du procédé, environ 1750 machines furent mises en service représentant 5500 lignes de coulées dans 90 pays, alors que la production mondiale d'acier doublait dans le même temps. Le taux d'équipement en coulée continue de la sidérurgie mondiale dépasse désormais $90 \%$. On a donc assisté à une véritable révolution industrielle affectant toute la profession sidérurgique, développement qui a 
fédéré d'énormes moyens en compétences et mobilisé des moyens financiers considérables. La coulée continue, au moins en ses débuts industriels, est surtout une affaire européenne et américaine et très vite japonaise, même si la diffusion vers les autres nations sidérurgiques sera rapide. La plupart des exemples que nous choisirons pour illustrer les évolutions techniques du procédé seront cependant majoritairement puisés dans les sidérurgies européennes (et plus particulièrement nationale), en raison de la connaissance plus complète que nous avons de leur histoire.

On ne saurait oublier que les développements en matière de $\mathrm{CC}$ ont été une contribution importante à la mise en oeuvre d'une «éco-filière de production de l'acier », ne serait-ce que par les économies d'énergie résultantes, même si ces préoccupations ne furent pas majeures dans les phases initiales de développement du procédé.

L'essentiel des technologies et des développements en matière cette coulée continue l'ont été au cours du demisiècle précédent (y compris l'avancée la plus innovante que fut la coulée de bandes), en conséquence on ne trouvera dans cet article à finalité d'histoire des techniques que peu de références postérieures aux années 1990.

\section{Partie I: les grandes étapes du développement de la coulée continue (CC)}

\subsection{La problématique coulée continue (CC)}

Pour planter le décor technique du développement historique du procédé et situer l'ampleur des problèmes qu'il convenait de résoudre (sur lesquels nous reviendrons en détail au cours de l'article), nous résumons ci-après les différentes composantes de la problématique à affronter par le sidérurgiste qui passe d'une coulée en lingots à l'exploitation d'une machine de coulée continue:

- le maintien en température (dans d'étroites limites au voisinage de $1500^{\circ} \mathrm{C}$ ) pendant toute la durée de l'opération, (qui peut dépasser l'heure pour une seule poche coulée) pour une masse d'acier liquide (de quelques dizaines de tonnes à quelques centaines de tonnes) contenue dans la poche de coulée.

- la tenue, sur le temps de coulée, des matériaux réfractaires constituant les canaux d'alimentation de la tête de machine et les dispositifs d'obturation;

- la régulation de débit de l'acier liquide afin de couler à vitesse constante, de conserver en machine un profil de solidification inchangé pendant la durée de coulée et surtout un niveau constant d'acier liquide en lingotière (forte incidence sur la qualité de peau);

- l'extraction régulière en continue du produit depuis la lingotière, sans endommagement de la peau du produit (lubrification), ni déchirures conduisants à la percée qui, si elle n'est pas toujours catastrophique, reste un incident sérieux;

- l'extraction thermique: évacuer dans les limites géométriques de la machine toute l'enthalpie de solidification de l'acier ;

- la qualité de peau du produit, qui doit être suffisante pour que celui-ci puisse être enfourné dans les fours de réchauffage du laminoir aval avec un minimum de parachèvement (voire à chaud sans aucun parachèvement), ce qui implique (entre autres exigences) une mâ̂trise très précise de la formation de la peau solide en lingotière ainsi qu'un soutien d'une grande précision du produit au long de la ligne de coulée;

- la santé interne du produit. Elle suppose non seulement d'éviter les endommagements internes (criques) résultant de contraintes excessives appliquées à un produit encore mal consolidé (dus, par exemple, à un désalignement de la machine), mais encore de minimiser les hétérogénéités compositionnelles et structurales issues $\mathrm{du}$ processus même de solidification, ainsi que les contaminations inclusionnaires [1]. Ces problèmes étaient d'autant plus aigus que le passage du lingot à la coulée continue diminuait le plus souvent fortement la section du produit solidifié; le travail de corroyage à chaud lors du laminage ultérieur (qui contribuait à atténuer les hétérogénéités issues du processus de solidification) serait donc bien plus limité sur un produit issu de $\mathrm{CC}$;

- une productivité et une disponibilité de la machine de coulée lui permettant de s'insérer harmonieusement entre les réacteurs de fusion/affinage et les laminoirs à chaud.

La figure 1 reproduit le schéma d'une coulée continue verticale pour brames. Le schéma coté permet d'apprécier l'encombrement de la machine.

À l'intention des lecteurs peu familiarisés avec les techniques sidérurgiques, l'annexe A regroupe quelques considérations élémentaires sur la conception et le fonctionnement des machines de coulée en usage dans la sidérurgie. On y trouvera notamment une ébauche de dimensionnement qui rend compte du grand développement linéaire des machines conventionnelles de coulée.

On peut s'interroger à propos des différences de vitesses de développement de la coulée continue dans l'industrie de l'aluminium (de l'ordre d'une ou deux décennies pour que la quasi-totalité de l'industrie de l'aluminium soit équipée) et dans la sidérurgie: près cent ans entre la première machine de $\mathrm{B}$. Atha chez Crucible Steel Co., en 1886 aux U.S.A. ans et la fin de la phase intensive de développement de la CC conventionnelle, et un peu plus de trente ans à partir d'une des premières machines réellement industrielles, celle de chez Böhler à Kapfenberg en Autriche en 1952.

Le tableau 1 qui regroupe quelques caractéristiques physiques et enthalpiques des deux matériaux (aciers et aluminium) rend compte pour partie non seulement des raisons à l'origine de la différence entre les vitesses de développement de la coulée continue respectivement dans les deux industries mais encore des différences considérables dans la complexité des machines mises en œuvre. Nous commentons ci-après ces différences de caractéristiques entre les deux matériaux:

- la solidification de l'acier se produit quelques $850^{\circ} \mathrm{C}$ audessus de celle de l'aluminium ce qui n'est pas sans conséquences sur le choix et la tenue des matériaux nécessaires à l'alimentation de la machine de coulée et au confinement de la solidification; 


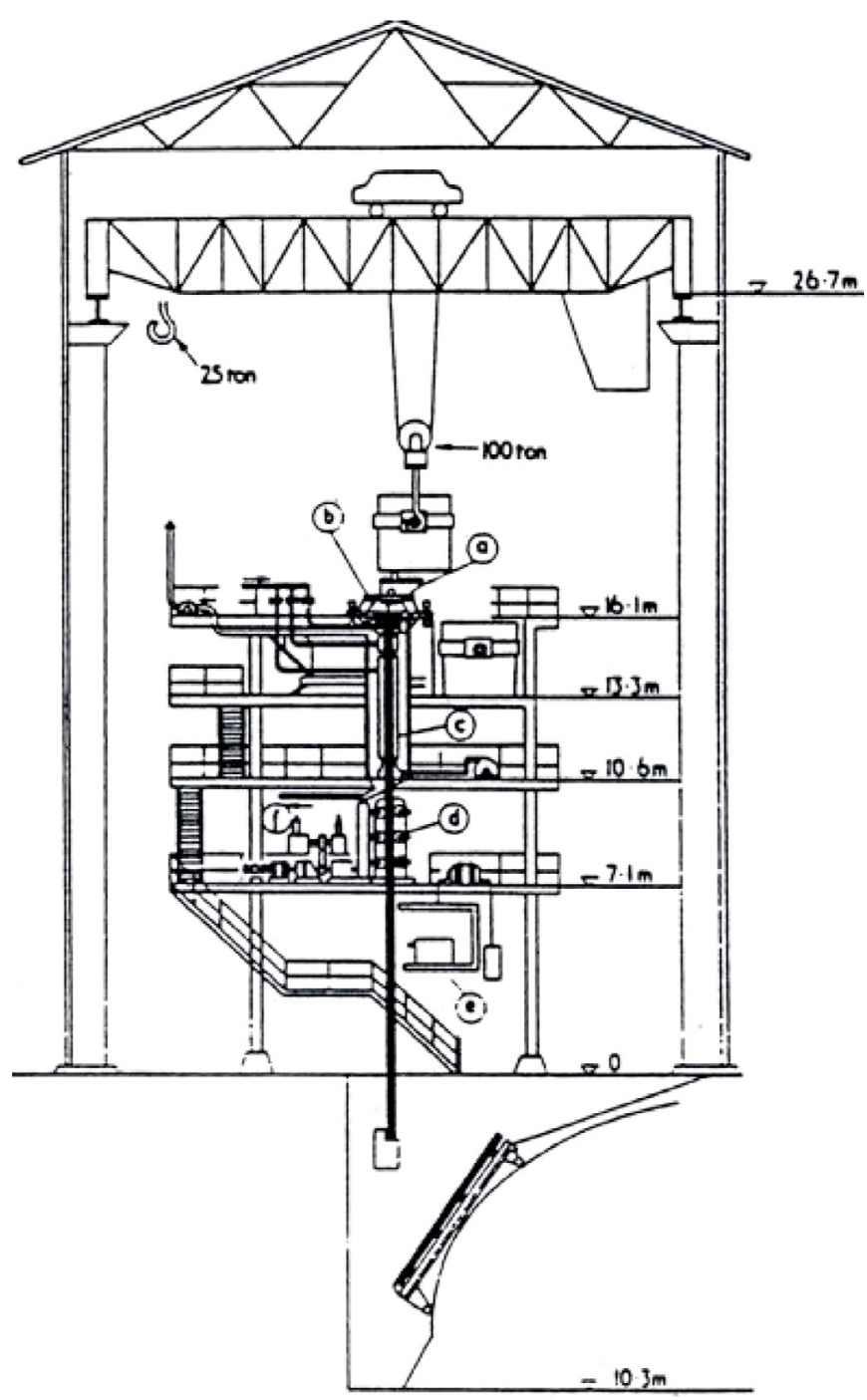

Fig. 1. Schéma d'une machine de coulée continue verticale [2-5]. Première machine pour larges brames en aciers inoxydables, construite en 1960 à l'usine de Hikari de Yawata Iron and Steel Co, ancêtre de Nippon Steel). a: Répartiteur; b: lingotière ; c : refroidissement secondaire; $d$ : extracteur ; e : coupe.

Fig. 1. Vertical casting machine.

- l'enthalpie à évacuer par unité de masse est, dans le cas de l'acier, de $10 \%$ supérieure à celle de l'aluminium;

- l'écart considérable de densité à l'état liquide (7,0 pour l'acier contre 2,4 pour l'aluminium) implique un corsetage de la machine (pour éviter les déformations en ligne), dans le cas de l'acier, sans commune mesure avec celui requis par l'aluminium. D'entrée de jeu, la machine de coulée continue pour l'acier ne pouvait être qu'une machine lourde, complexe, devant opérer en milieu sidérurgique (qui n'est pas précisément proche de celui d'un laboratoire);

- la conductivité thermique de l'aluminium solide bien plus élevée que celle de l'acier permet une extraction thermique en machine de coulée bien plus efficace et permet donc un raccourcissement notable des lignes de coulée de l'aluminium. On notera au passage la faible conductivité de l'austénite alliée comparée à celle de l'acier au carbone. Ce ne sera pas sans conséquences sur la coulée des aciers inoxydables austénitiques;

- la complexité, dans le cas des aciers, des phénomènes métallurgiques à la solidification et au refroidissement depuis le solidus (précipitations diverses, transformations de phases) ayant un grand retentissement sur la qualité du produit final impliquait que le passage à la coulée continue, pour les nuances les plus complexes, ne se fasse qu'après l'élucidation complète de ces phénomènes et la mise au point de mesures palliatives à leurs effets éventuellement nocifs sur la qualité. Cette élucidation ne pouvait se faire que de manière très progressive, au rythme de l'accroissement des connaissances à propos des phénomènes physico-chimiques en jeu sur la machine et du retour d'expérience des machines industrielles ;

- la considérable variété des applications mécaniques de l'acier impliquait que chacune des industries clientes valide, pour son propre compte, la nouvelle filière de coulée en évaluant le matériau coulé pour des propriétés aussi diverses que l'isotropie et l'homogénéité des propriétés mécaniques, le comportement en endurance, l'aptitude à la déformation à froid, l'aptitude aux traitements de surface, la tenue à la corrosion...

\subsection{Pourquoi la coulée continue?}

Les ingénieurs en charge du développement de procédés de fabrication ont toujours été séduits par les procédés continus, qui comparativement aux procédés discontinus, se prêtent en général beaucoup mieux à l'instrumentation, à l'automatisation, aux gains de productivité. Ces procédés continus suppriment, en outre, une bonne partie des régimes transitoires à l'origine de beaucoup de problèmes qualitatifs. La sidérurgie ne pouvait rester à l'écart d'une telle évolution, notamment parce que le bassin de coulée en lingots restait le maillon faible de l'aciérie par son caractère encore largement artisanal, le faible contrôle que l'on y avait sur les processus, et les sévères conditions de travail qui y régnaient.

Mais la vraie justification technico-économique de la coulée continue était d'obtenir une ébauche brute de coulée au format le plus proche possible de celui du produit final sortant de l'usine sidérurgique, afin de minimiser le travail de laminage à chaud et en particulier de court-circuiter les cages dégrossisseuses imposées par le format des lingots, ce qui engendrait une simplification très notable des gammes de laminage. En effet, la coulée en lingots imposait presque systématiquement un laminage «en deux chaudes»: le produit (bloom ou brame) sortant du laminoir dégrossisseur (blooming pour les produits longs, slabbing pour les produits plats) devait être réchauffé une seconde fois avant d'entrer dans un train finisseur (train à barres, train à fils, train continu à bandes). Grâce à la coulée continue, on passait ainsi de lingots de poids unitaires de quelques tonnes et dont la section droite pouvait être de plusieurs dizaines de $\mathrm{dm}^{2}$ (lingots carrés pour les produits longs et lingots méplats pour les produits plats) à des produits de $\mathrm{CC}$ dont les sections typiques étaient $120 \times 120 \mathrm{~mm}^{2}$ pour 
Table 1. Caractéristiques physiques et enthalpiques comparées des aciers et de l'aluminium [6].

Table 1. Physical and thermochemical properties of steels and aluminium.

\begin{tabular}{llll}
\hline & Aciers bas carbone & $\begin{array}{l}\text { Acier inox } \\
\text { austénitique }\end{array}$ & Aluminium \\
\hline$T$ de fusion $\left({ }^{\circ} \mathrm{C}\right)$ & 1535 & $1400 / 1450$ & 660 \\
$\begin{array}{l}\text { Enthalpie à la } T \text { de fusion, } \\
\text { (enthalpie de fusion incluse) }\end{array}$ & 0,364 & 0,342 & 0,325 \\
$(\mathrm{Kwh} / \mathrm{Kg})$ & & & \\
$\begin{array}{l}\text { Densité à l'état liquide }\left(\mathrm{g} \cdot \mathrm{cm}^{-3}\right) \\
\begin{array}{l}\text { Conductivité thermique } \\
\left(\mathrm{Wm}^{-2} \mathrm{~K}\right)\end{array}\end{array}$ & 7,0 & 7,0 & 2,36 \\
\hline
\end{tabular}

Table 2. Besoins en énergie (kwh/t de semi-produit) de la coulée continue et de la coulée en lingots [2-5].

Table 2. Energy consumption ( $k w h / t$ of semi product) in continuous casting and ingot casting.

\begin{tabular}{lcll}
\hline Coulée continue & \multicolumn{3}{c}{ Coulée en lingots } \\
\hline Surchauffe de l'acier liquide & 10 & Énergie électrique des fours & 6,0 \\
Préchauffage du répartiteur & 35 & Combustible pour le réchauffage & 290,6 \\
& & des lingots (50\% de lingots enfournés froids) & 20 \\
Énergie électrique/machine de coulée & 18 & Énergie électrique du laminoir & 316,6 \\
Total & 63 & Total &
\end{tabular}

des billettes destinées à un train à fil ou pour ronds à béton, de $250 \times 250 \mathrm{~mm}^{2}$ pour des blooms destinés à la production de barres en aciers de construction, et de $250 \times 1800 \mathrm{~mm}^{2}$ pour des brames destinées aux produits plats. Bien entendu, en fonction du carnet de commandes et des exigences qualitatives qu'il contenait, de la prise de fer du laminoir aval, les sidérurgistes pouvaient s'écarter notablement des formats typiques que nous venons d'indiquer. Nous conserverons la classification des machines de coulée en trois grandes catégories: les machines à billettes pour des formats coulés inférieurs à $180 \times 180 \mathrm{~mm}^{2}$, les machines à blooms pour des formats en général inférieurs à $400 \times 400 \mathrm{~mm}^{2}$, les machines à brames pour des formats autour de $250 \times 1800 \mathrm{~mm}^{2}$, formats auxquels s'ajoutent les ébauches pour rails et profilés divers. D'une manière générale, plus les exigences qualitatives en matière de santé interne étaient sévères plus on avait tendance à faire grossir le format choisi de manière à ce que le travail de corroyage en aval, au cours du laminage, reste suffisamment important pour atténuer les hétérogénéités issues du processus de solidification; mais évidemment plus le produit grossissait plus la machine de coulée s'alourdissait. Certains sidérurgistes seront amenés à concevoir des machines coulant de quasi-lingots (le japonais Sanyo Steel Co sera l'un des premiers à couler en continu, au format $370 \times 480 \mathrm{~mm}^{2}$, avec plein succès, des aciers à roulements ; Timken construira dans son usine de Faircrest (USA) une machine $\mathrm{CC}$ au format $460 \times 610 \mathrm{~mm}^{2}$ pour ces mêmes aciers); mais avec de tels formats on n'obtenait pas la simplification des gammes de laminage à chaud que des formats notablement plus petits permettaient.
La crise pétrolière des années 1973/1974 accéléra de par le monde le passage en coulée continue puisque l'élimination d'une chaude de laminage (blooming ou slabbing) entraînait une économie d'énergie fort significative (Tab. 2). C'est à partir des années 1970 que la coulée continue prit vraiment son essor en même temps que la production mondiale d'acier croissait fortement (Fig. 2) principalement sous l'impulsion de la Chine qui deviendrait le premier producteur mondial. La part de la coulée continue dans la production mondiale d'acier était de $4 \%$ en 1970 , de $25 \%$ en 1980 , de $60 \%$ en 1990 , et de plus de $90 \%$ actuellement. Le formidable développement de la Chine en matière de sidérurgie (sa production représente désormais $50 \%$ de la production mondiale) s'est fait essentiellement à partir de coulées continues. Entre temps, certains problèmes majeurs avaient été résolus et la technologie largement fixée (nous y reviendrons en détail dans le cours de cet article). L'industrie sidérurgique tirait enfin (à partir des années 1970) le bénéfice des vingt années précédentes d'efforts intensifs de développement.

Nombre d'usines sidérurgiques pousseront la recherche des économies d'énergie jusqu'à procéder à l'enfournement chaud, dans les fours de réchauffage des laminoirs, des produits sortant de la CC. Le procédé n'était cependant pas généralisable à toutes les nuances d'acier notamment parce que la qualité de surface du produit en sortie machine imposait parfois un parachèvement avant enfournement pour laminage à chaud.

La simplification des gammes de laminage résultant du passage en coulée continue avait aussi des retombées favorables sur les coûts de fabrication, au-delà des économies d'énergie mentionnées ci-dessus : 


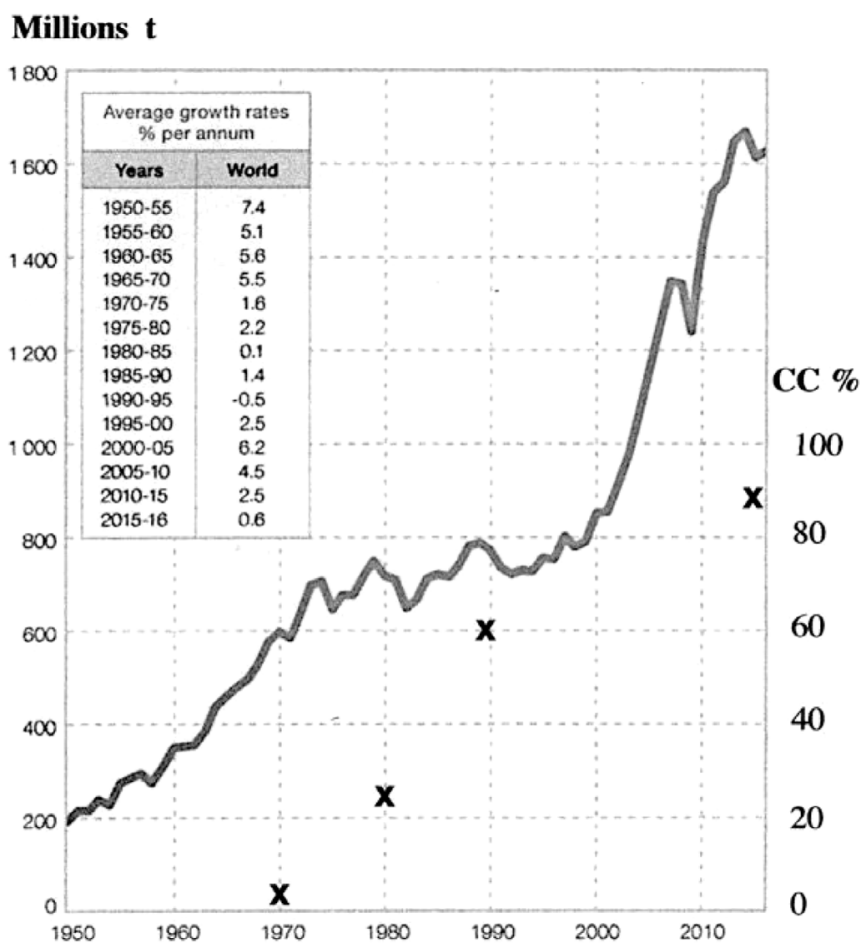

Fig. 2. Évolution de la production mondiale d'acier et part de la coulée continue $(\mathbf{X})$ [7].

Fig. 2. World steel production evolution and part of continuous casting $(\boldsymbol{X})$.

- un gain sensible sur les rendements métalliques, en réduisant les pertes de métal par oxydation (calamine) du fait de la suppression d'une chaude de laminage et surtout les chutages au cours de la mise en forme. En voie lingot on procédait, après les premières passes de laminage, à un double chutage (tête et pieds) du produit issu de chaque lingot; en CC on ne procédait à un tel chutage que pour chacune des lignes de coulée démarrées, c'est-à-dire de la coulée sans interruption de l'extraction. Ce gain croissait bien évidemment avec la longueur des séquences de coulée;

- simplification des manutentions ;

- réduction des délais de fabrication.

\subsection{Les grandes étapes du développement de la coulée continue}

Le Dr Wolf distingue six périodes dans le développement de la coulée continue appliquée aux aciers [2-5].

\subsection{1 《La période préhistorique »}

«La période préhistorique » qui s'étend de 1886 (première machine d' Ath a chez Crucible Steel U.S.A.) jusqu'au début des années 1940. La machine d'Atha (Fig. 3) est verticale, à lingotière fixe, à paroi épaisse en fonte (donc comparable à une lingotière classique de la voie lingot) et à extraction discontinue. On mesure tout le chemin qui reste à parcourir

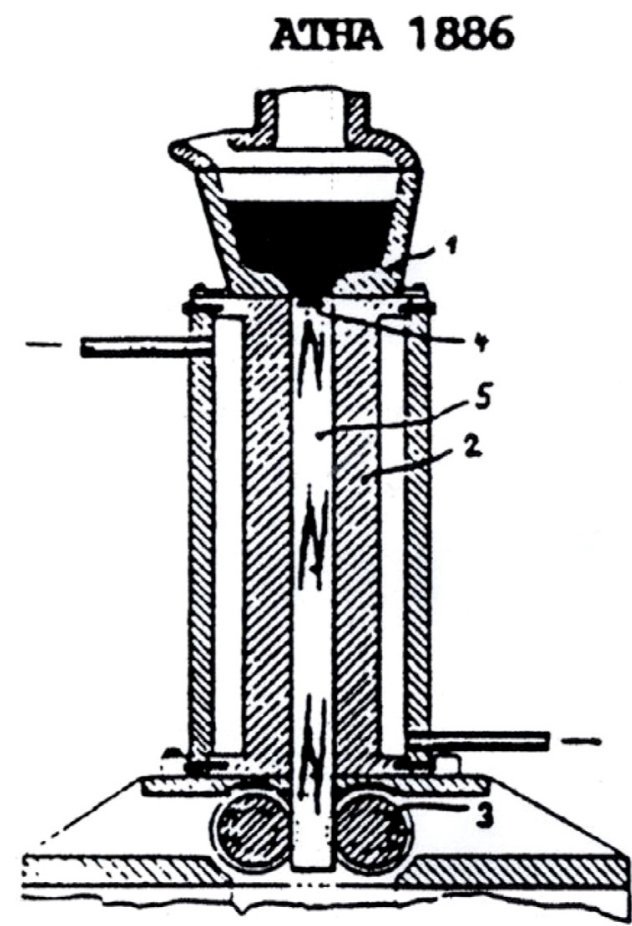

Fig. 3. Première machine de coulée continue (B. ATHA: Crucible-Steel Co 1886) [2-5].

Fig. 3. First continuous caster (B. Atha-1886).

puisque les machines qui s'imposeront dans l'industrie seront à lingotière à paroi mince, oscillante, à extraction continue, et souvent courbes. En attendant les premières machines se complexifient sur la base de développements empiriques puisqu'on ne dispose pas des connaissances suffisamment précises en matière de solidification des aciers pour orienter rationnellement les développements ; la place de la CC dans les aciéries reste confidentielle. La coulée continue des métaux non ferreux apparaît, se développe rapidement, et inspire les développements en matière de coulée des aciers. C'est au cours de cette période (années 1930) qu'apparaissent les technologies de base pour la lingotière de coulée continue: la paroi mince, l'oscillation verticale, la lubrification. Le nom de S. Junghans (18871954) reste associé à cette période pionnière.

\subsubsection{Les années $1940:$ L'acier peut-il vraiment être coulé} en continu? »

Pendant la guerre et immédiatement après celle-ci, des essais sont menés un peu partout dans les pays industrialisés et notamment en Allemagne (toujours par Junghans), aux USA (Bethleem Steel, Republic Steel, Babcock \& Wilcox), en URSS, en G.B. Dès 1947, des essais sont repris par Junghans en Autriche avec une collaboration des sociétés Böhler, Schöller-Bleckmann et VoestAlpine et au Japon. Nous nous arrêterons un court instant sur ce dernier pays: Sumitomo construit en 1947 à Amagasaki une machine verticale pour couler des ébauches 

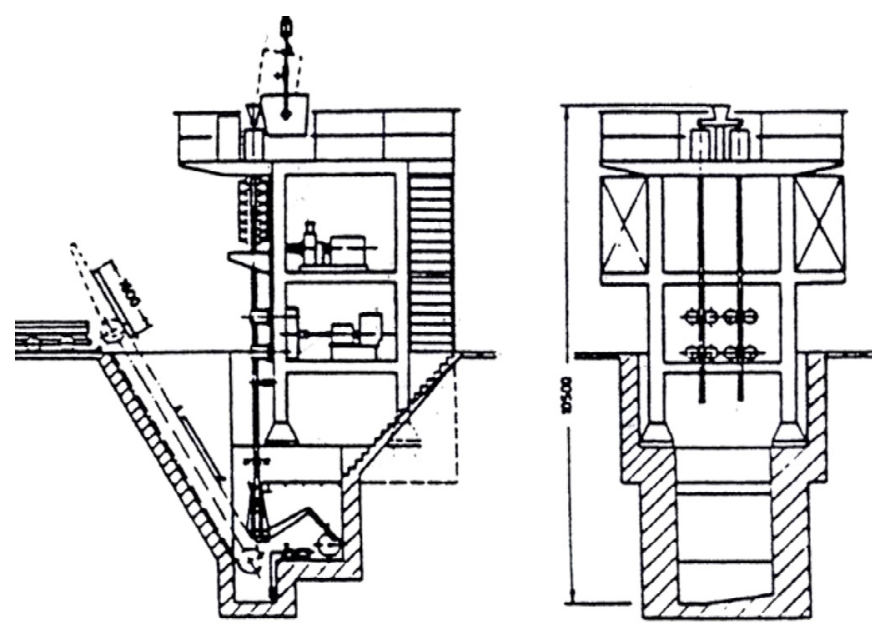

Fig. 4. Première machine industrielle de Junghaus pour minibrames $\left(100-160 \times 330-100 \mathrm{~mm}^{2}\right)$ et billettes $\left(90\right.$ à $\left.140 \mathrm{~mm}^{2}\right)$ chez Böhler/Kapfenberg (1952) [2-5].

Fig. 4. Vertical billet and minislab caster (Böhler/Kapfenberg 1952).

pleines pour tubes sans soudure. Quand on imagine l'état de l'industrie japonaise deux ans après la fin de la guerre, la performance paraît assez exceptionnelle. Revenons aux développements en Autriche: la machine à deux lignes construite par Junghaus chez Böhler à Kapfenberg (Fig. 4) constituera un véritable banc d'essai pour la technologie naissante, par la diversité des formats coulés (mini-brames, billettes rondes et carrées), des nuances (y compris des aciers inoxydables); on y conduira même des essais de cintrage pour ouvrir la voie aux machines courbes. C'est dans cette période que les technologies de base apparues dans la période précédente se fixent et qu'apparaissent aussi les toutes premières tentatives d'instrumentations de la tête de machine avec l'emploi des rayons $\mathrm{X}$ pour repérer le niveau de métal en lingotière et un moniteur TV visant le ménisque (B\&W à Beaver Fall, U.S.A.), le four à induction en tête de machine pour maintenir le métal à température, le répartiteur pour alimenter simultanément plusieurs lignes de coulée. On s'étonnera peut-être de la détermination des sidérurgistes à développer une nouvelle technologie, alors qu'au sortir de la guerre tant d'usines étaient à reconstruire (c'était particulièrement vrai pour le Japon et l'Allemagne), mais de grands industriels visionnaires commençaient à rassembler des équipes d'ingénieurs constructeurs, exploitants, chercheurs, car ils avaient parfaitement compris qu'il valait mieux reconstruire des usines sur la base des technologies de demain plutôt que sur celle des technologies d'avantguerre.

\subsubsection{Les années 1950 : les premières exploitations des machines industrielles}

Le début des années 1950 est marqué par la naissance des premières sociétés d'engineering se consacrant à la conception des machines de coulée continue. Deux personnalités visionnaires et emblématiques, qui bien qu'engagées dans des entreprises concurrentes resteront en contacts techniques étroits, vont jouer un rôle capital dans ce développement: S. Junghans que nous avons déjà rencontré dans cette histoire et I. Rossi (1889-1991). Le premier se rapproche du sidérurgiste Mannesmann avec la construction en 1954 de la CC1 d'Huckingen (machine à blooms verticale à 4 lignes). Le second fonde en 1950 (à l'âge où l'on prend d'ordinaire sa retraite) aux USA une société d'engineering (Continuous Métalcast) qui devait donner naissance en Suisse à CONCAST AG en 1954 avec l'ambition de développer une machine à forte productivité et donc à grande vitesse d'extraction, dont il pensait avec lucidité qu'elle aurait un grand avenir dans un monde sidérurgique largement en reconstruction. Les deux hommes manifestaient une égale détermination à développer la coulée continue des aciers après avoir fait leurs premières armes dans les machines de coulée pour métaux non ferreux. Ainsi se mettaient en place deux pôles majeurs de développement pour la CC avec deux groupements constructeurs leaders pilotés chacun par l'un des deux pionniers (mais S. Junghans décèdera en 1954) :

- le premier autour de l'association Junghans/Mannesmann s'appuyant aussi sur l'expérience de Junghans chez BÖHLER. Ce groupe sera rejoint par DEMAG qui apportera sa vaste expérience dans la construction des usines sidérurgiques ;

- le second autour de CONCAST qui fédère les participations financières de BSC (British Steel Corporation), de $\mathrm{GHH}$ et du groupe Marine-Firminy, met en commun l'expérience acquise sur leurs territoires par ces partenaires et promeut la coopération des engineerings associés (pour la France CAFL Engineering qui deviendra CLECIM). Ce groupement sera rejoint par SUMITOMO (SHI) ; entre-temps SUMITOMO-METAL aura démarré en 1955 à Osaka la seconde CC du groupe après celle d'Amagasaki.

Ces deux groupes constructeurs concurrents conserveront cependant un point commun avec MBC (Mannesmann, Böhler, Concast).

Si l'on considère aussi la forte présence de CONCAST aux USA (Concast Inc) et ses autres développements de par le monde (Inde, Espagne...) ainsi que les développements propres des constructeurs japonais, US ou de l'URSS, on peut considérer que l'internationalisation de la technologie était en bonne voie ; elle était bien la conséquence du très vif intérêt des aciéristes exploitants pour le procédé.

On trouvera regroupés au tableau 3, les noms des principales sociétés d'engineering (sans aucune prétention à l'exhaustivité) qui contribuèrent de manière très significative au développement mondial de la coulée continue dans les aciéries. La filiation de ces entreprises est compliquée car comme pour toute technologie émergeante, au marché fortement croissant, le nombre d'acteurs commence par augmenter fortement pour aboutir ensuite à des rationalisations. Elles se rattachent bien souvent, plus ou moins, par le jeu des licences ou des accords successifs, aux deux pôles constructeurs initiaux évoqués précédemment. Quelques «indépendants» des deux groupements fondateurs n'en ont pas moins joué un rôle très significatif dans le développement: Fives-Cail-Babcock (FCB) en France, Voest-Alpine Industrialbau (VAI) en Autriche, 
Table 3. Principaux constructeurs de machines de coulée continue dans la phase intensive de développement.

Table 3. Main constructors of CC machines during the intensive development period.

\begin{tabular}{lll}
\hline Sociétés & Nationalité & Remarques \\
\hline CONCAST & Suisse & Extension mondiale des partenariats \\
VOEST-ALPINE (VAI) & Autriche & \\
FIVES-CAIL-BABCOCK (FCB) & France & Proche de Demag et partenaire important d'Usinor \\
$\begin{array}{l}\text { DUJARDIN-MONTBARD- } \\
\text { SOMENOR (DMS) }\end{array}$ & France & $\begin{array}{l}\text { Constructeur des machines rotatives } \\
\text { (proche d'Imphy et de Vallourec) }\end{array}$ \\
$\begin{array}{l}\text { CLECIM } \\
\text { (CAFL Engineering, initialement) }\end{array}$ & France & Partenariat avec CONCAST \\
$\begin{array}{l}\text { SUMITOMO HEAVY } \\
\text { INDUSTRIES (SHI) }\end{array}$ & Japon & Idem \\
HITACHI-ZOSEN & & Partenariat avec DEMAG \\
MANNESMANN-DEMAG (MDM) & Allemagne & Extension mondiale des partenariats \\
SCHLOEMANN- & Japon & Partenariat avec CONCAST \\
DEMAG-SIEMAG (SMS) & Allemagne & \\
DISTINGTON/DAVY & & Partenarit avec CONCAST \\
DANIELI & G.B. & \\
KOPPERS & Italie & \\
ROKOP & U.S.A. & \\
\hline
\end{tabular}

Danieli en Italie, Davy en G.B, Koppers et Rokop aux USA. Bon nombre des constructeurs regroupés au tableau 3 ont soit disparu, soit ont été incorporés dans des groupes de mécaniciens/engineering plus généralistes.

La contribution de l'URSS (non mentionnée au Tab. 3) au développement du procédé fut très significative, si l'on en juge par les installations construites dans les territoires sous contrôle soviétique, souvent avec des conceptions technologiques très originales. Un exemple de cette originalité est fourni par la machine de Nijny-Taguil (Oural) démarrée en 1968. Il s'agit d'une machine courbe à brames, à refroidissement secondaire par jeux de longerons et walking-beams. Nous reviendrons sur cette technologie particulière dans la partie II [15].

En France en 1953, sous l'impulsion d'H. Malcor, une machine de coulée démarre à Unieux dans la Loire (future usine CAFL) en petits formats, elle coulera, dans des formats ovales, une grande variété de nuances, y compris des aciers à outils, ce qui était un vrai défi pour l'époque. On notera que le démarrage de cette machine se place dans la période de construction des quatre premières machines de Junghans. H.
Malcor est aussi à l'origine de la construction en 1955 de la machine à billettes d'Allevard coulant des aciers à ressorts (aciers au silicium). Cette machine sera suivie en 1963, dans la même aciérie, d'un second équipement, ce qui démontrait la pleine adéquation de la coulée continue à la production des aciers faiblement alliés en produits longs. H. Malcor sera un acteur important dans le développement de CONCAST et de CAFL Engineering devenu CLECIM.

Fives-Cail-Babcock (FCB) fait en 1954 ses premiers pas en matière de $\mathrm{CC}$ avec la coulée à blooms à quatre lignes verticales chez Usinor/Denain.

La production mondiale d'acier double presque dans cette décennie pour atteindre $340 \mathrm{Mt}$ en 1960 , mais elle est encore dépendante à $80 \%$ des fours Martin qui se prêtent mal (longueur de l'élaboration, fort tonnages unitaires coulés) à la coulée continue, telle qu'elle émerge de ses premiers pas vraiment industriels. Celle-là se développe donc plutôt en aval de fours électriques qui sont loin d'avoir atteint leurs pleines performances [8-10] et restent encore le plus souvent de tonnages limités $(<30 \mathrm{t})$. Ce sont donc le plus souvent des aciéries pour produits longs (souvent en 


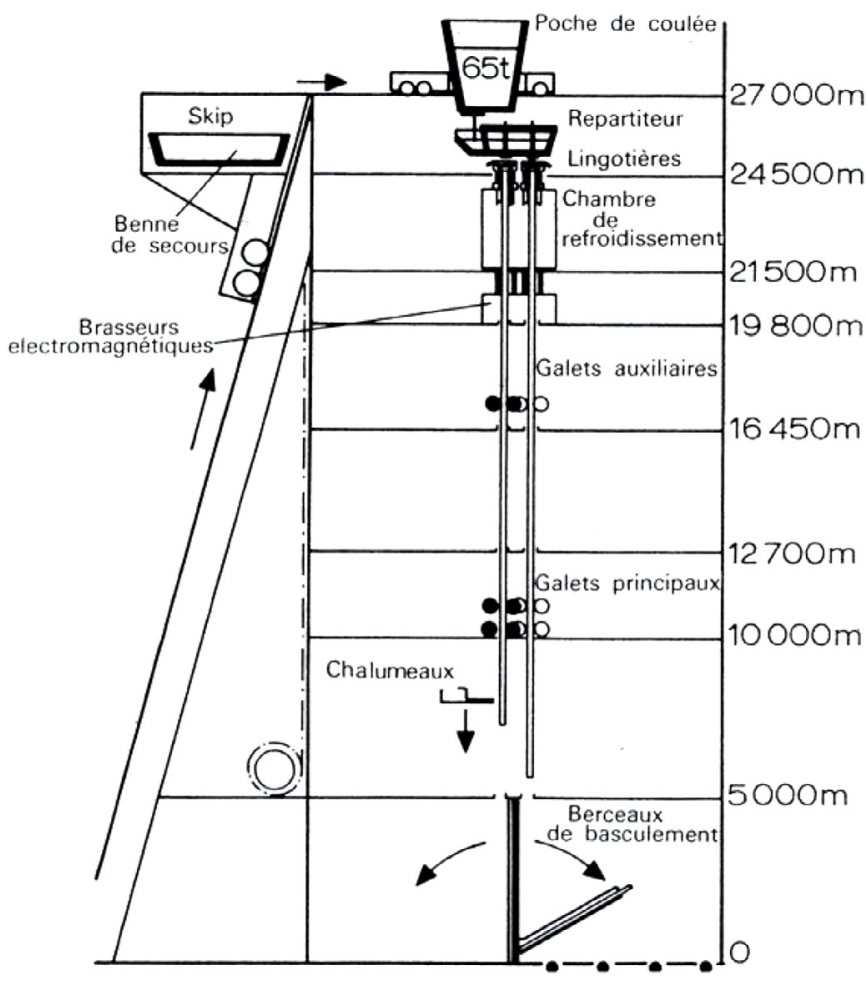

Fig. 5. La machine verticale à petits blooms de la SAFE (1960) [11].

Fig. 5. Small bloom vertical caster at SAFE.

aciers spéciaux faiblement alliés ou pour le rond à béton), pratiquant majoritairement la fusion électrique, qui s'équipent de machines à blooms ou à billettes. On notera l'équipement dès 1960 de l'aciérie d'Hagondange (SAFE, appartenant alors à Renault; l'usine appartient aujourd'hui à Asco-Industries) avec une machine verticale à 4 lignes coulant des billettes de $120 \times 120 \mathrm{~mm}^{2}$ et des blooms jusqu'à $200 \times 200 \mathrm{~mm}^{2}$ en aciers de construction faiblement alliés destinés à l'industrie automobile (Fig. 5) (cette machine sera reconstruite en 1986 par l'engineering FCB puis modernisée en 1991 pour couler toujours suivant la verticale des blooms de $240 \times 240 \mathrm{~mm}^{2}$ ).

La configuration des quatre lignes de coulée de la première machine de la SAFE est singulière puisqu'elles sont disposées en carré et non en ligne comme cela deviendra la règle (cela démontre qu'on a mené une réflexion originale sur l'alimentation de la machine). Il est intéressant de noter que le constructeur de cette machine fut le consortium (évoquécidessus) formé autour de Mannesmann, Böhler, et Junghans, l'un des principaux développeurs à l'époque de la technologie de CC. Une machine verticale à blooms à quatre lignes est aussi construite chez Mannesmann/Hückingen en Allemagne (Fig. 6). C'est dans cette période que se généralise l'oscillation sinusoïdale de la lingotière. Ces machines sont verticales (pour la partie active en solidification), mais le cintrage apparaît déjà en sortie de machine (pour évacuer le produit à l'horizontale, augmenter la longueur des lignes et donc couler plus vite); cependant nombre de machines restent totalement verticales avec coupe du produit en position verticale et remontée des blooms par un skip (cas de la SAFE ou de Mannesmann/Hückingen).

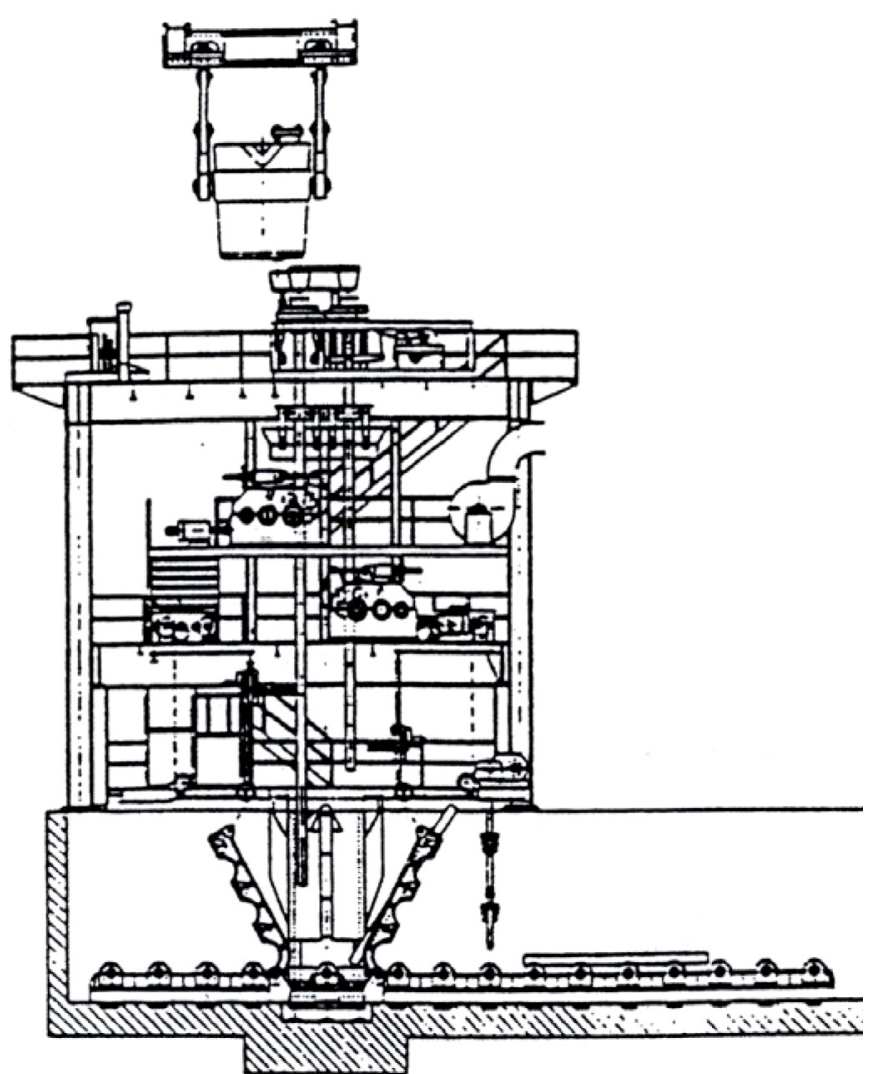

Fig. 6. Coulée continue verticale de blooms à 4 lignes chez Mannesmann/Hückingen (1954) [2-5].

Fig. 6. Vertical Bloom caster at Mannesmann Hückingen (1954).

On signalera la construction en 1954, chez ATLAS STEEL à Weiland (Canada), d'une machine verticale mixte billettes $\left(140 \times 190 \mathrm{~mm}^{2}\right)$ et larges blooms $(100-165$ $\times 483-610$ ) coulant des aciers inoxydables. La machine construite par KOPPERS avait été conçue par I. Rossi.

\subsubsection{Les années 1960 : la révolution apportée par la machine à lingotière courbe}

Plus le produit augmentait en section, plus la longueur en machine nécessaire à sa solidification complète croissait. Cela conduisait à des hauteurs de machines verticales le plus souvent incompatibles avec les aciéries existantes puisqu'elles ne disposaient pas des hauteurs suffisantes sous crochet de pont roulant pour amener la poche d'acier liquide en tête de machine de coulée. Il n'y avait que deux solutions au problème posé : enterrer la sortie de machine au fond d'un «gouffre de Padirac » (solution retenue par la SAFE), ou construire une halle lourde spécifique pour abriter la coulée continue, mais conçue pour manipuler des charges de plusieurs dizaines et même centaines de tonnes à grande hauteur, avec le surcoût considérable que cela représentait au point de vue des structures porteuses du bâtiment. Une première approche de solution au problème de hauteur consistait à courber le produit dans sa partie aval, puis à le redresser avant la coupe, mais avec l'exigence que la solidification soit terminée avant le cintrage qui devait donc être positionné assez bas dans la machine. Ce 


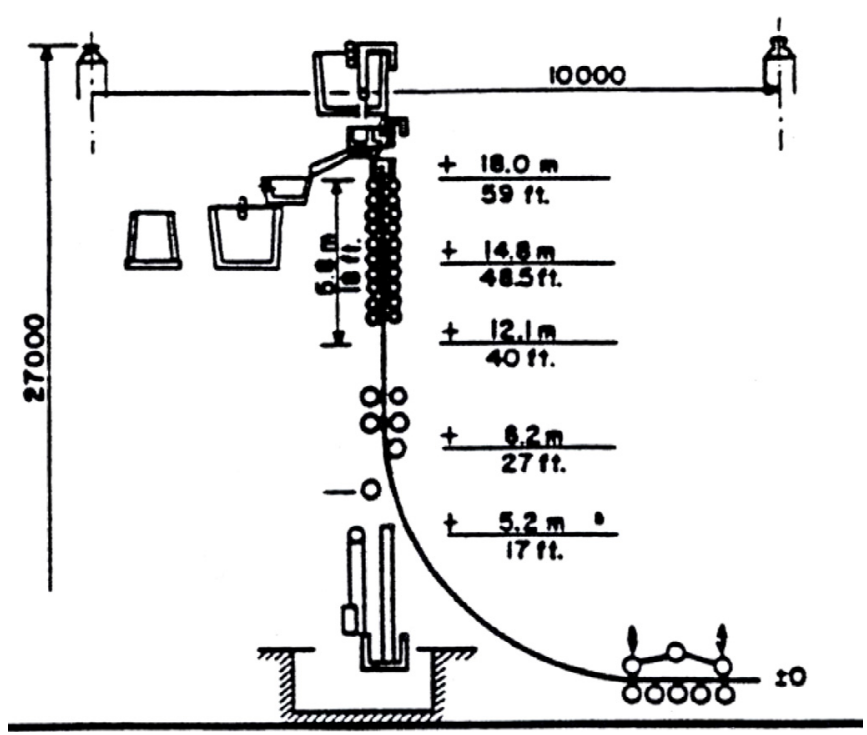

Fig. 7. Machine verticale avec cintrage pour larges brames à Dillingen (1961) [2-5].

Fig. 7. Vertical/bending wide slabs caster at Dillingen (1961).

sera la solution adoptée en 1961 à Dilling en Sarre pour couler de larges brames $(200 \times 800 / 1000 / 1520)$, (Fig. 7). On notera que la lingotière se situe, malgré la courbure de la ligne, à $18 \mathrm{~m}$ du sol et le chemin de roulement du pont de coulée à $27 \mathrm{~m}$ ! Les machines suivantes de Dilling seront construites suivant ce schéma, c'est-à-dire avec un profil vertical droit suivi d'un cintrage sur cœur solide (Fig. 8) puis d'un décintrage.

La conception d'une machine avec lingotière courbe prolongée par un refroidissement secondaire développé sur un presque quart de cercle de même rayon, résolvait pour partie le problème du développement en hauteur. Il fallait cependant décintrer le produit, un peu avant sa sortie de la machine pour évacuer à l'horizontale un produit devenu rectiligne. Cela poserait d'autres problèmes (nous y reviendrons). La première machine pilote de coulée de billettes $\left(85 \times 85 \mathrm{~mm}^{2}\right)$ à lingotière courbe sera construite en 1963 chez Von Moos à Lucerne (Fig. 9). Elle sera suivie en 1964 par la machine à lingotière courbe pour billettes et minibrames chez Bohler/Kapfenberg (Junghaus) remplaçant l'ancienne machine verticale. Une des premières machines à lingotière courbe pour larges brames sera construite en 1964 par Concast/Schloemann pour Dillinger-Hütte (rayon $8 \mathrm{~m}, 1$ ligne $250 \times 1550 \mathrm{~mm}^{2}$ ) (Fig. 8), elle sera suivie de beaucoup d'autres de par le monde... C'est la lingotière courbe qui débloque vraiment la coulée continue pour les produits plats avec les machines à brames. Nous retiendrons les premières machines à brames au Japon en 1967 chez Yamato Steel et NKK, prémisses d'un futur et magnifique développement. C'est sur la machine de NKK que sera établi le premier record significatif de coulées en séquence avec 28 coulées (1970). On notera l'entrée de la Chine dans le club de la CC pour brames avec la machine de Chongquing/Sechuan (rayon $6 \mathrm{~m}, 1$ ligne au format $\left.200 \times 1700 \mathrm{~mm}^{2}\right)$.
C'est aussi dans cette période qu'on observe le développement du phénomène «Bresciani» des miniusines basées sur la fusion de ferrailles au four électrique pour la production de produits longs en nuances courantes (ronds à béton, fers marchands, fils...). Le phénomène n'aurait pu prendre l'ampleur qu'il connut sans l'existence de la coulée continue à billettes. Les deux principaux constructeurs en Europe de ce type de machine seront CONCAST et DANIELI. Le choix est fait d'une machine entièrement courbe, modulaire, dépouillée au maximum, coulant à grande vitesse. La production visée est alors de 25000 t par ligne et par an. Ces machines représentent la réalisation parfaite de l'objectif de Rossi lorsqu'il se lança dans la coulée continue: une machine simple et de haute productivité. Les Bresciani (dont le groupe Riva) resteront comme les inventeurs du modèle de l'aciérie de haute productivité, centrée sur le four électrique amené à de hautes performances [8-10] couplé à une coulée continue de format limité coulant à grande vitesse.

\subsubsection{Le développement massif de la coulée continue} (les années 1970)

Nous avons déjà signalé que la crise pétrolière va donner une impulsion décisive au développement de la $\mathrm{CC}$ en valorisant grandement les économies d'énergie issues de la suppression d'une chaude de laminage. Le développement est particulièrement significatif pour les produits plats et les machines à brames. À partir d'une technologie désormais mature, le Japon mène la course au développement notamment en produits plats: augmentation des vitesses de coulée, amélioration des qualités, extension du carnet des nuances coulables en continue (aciers inoxydables en particulier), amélioration de la tenue des réfractaires de coulée, généralisation de la busette à tiroir pour contrôler le débit d'acier, généralisation des busettes à ouïes pour alimenter la lingotière, instrumentation et automatisation des fonctions autour de la tête de machine (Fig. 10), coulées en longues séquences.

La sidérurgie française des produits plats s'équipe en machines à brames à partir de 1968 (Tab. 4).

On trouvera au tableau 5, à la fin de cet aperçu historique, les principaux sites sidérurgiques français (liste non exhaustive) qui s'équipèrent de machines de coulée continue. Ce tableau est aussi un reflet assez fidèle des restructurations ayant affecté la sidérurgie française (par le nombre d'installations arrêtées qui y figurent). On notera aussi que le développement des machines à brames est décalé d'environ dix ans par rapport à celui des machines à blooms et à billettes, ce qui traduit non seulement la différence de complexité entre les deux types de machines et la plus grande difficulté à maîtriser l'exploitation des premières, mais encore l'enjeu financier et technique considérable consistant à arrêter dans une usine intégrée à produits plats le laminoir slabbing, ces risques ne pouvant être valablement assumés qu'à partir d'une technologie de $\mathrm{CC}$ parfaitement fiable. Au regard des enjeux financiers et commerciaux, la lucidité des exploitants de CC brames a été l'une des clés du succès. 


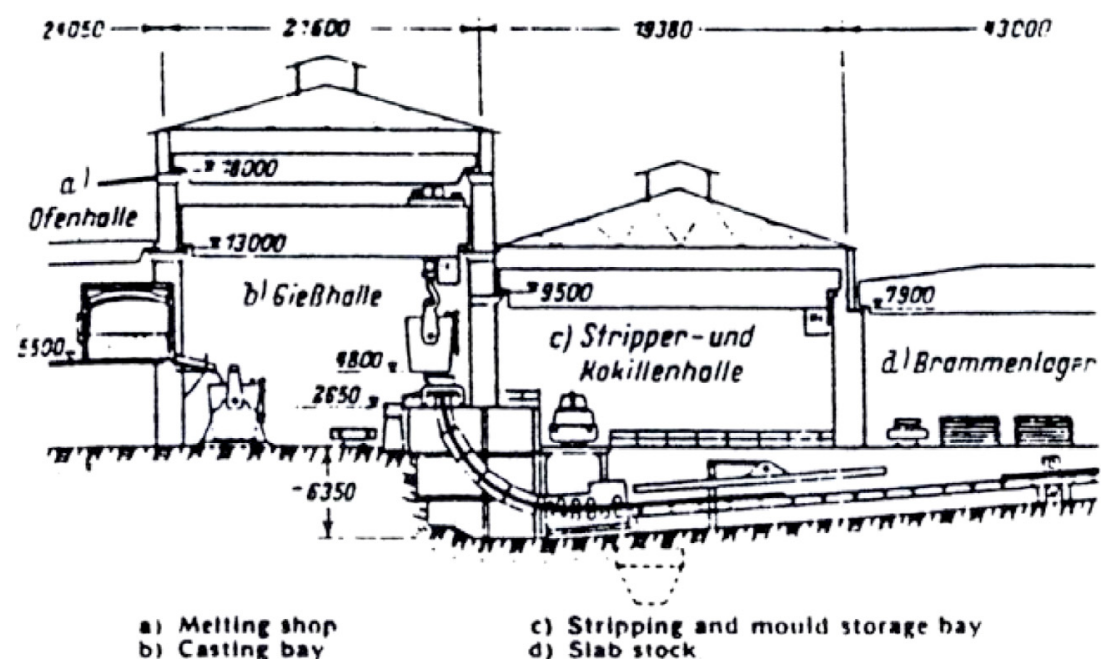

Fig. 8. Machine à lingotière courbe pour larges brames à Dillingen (1964) [2-5].

Fig. 8. Curved mold caster for wide slabs at Dillingen (1964).

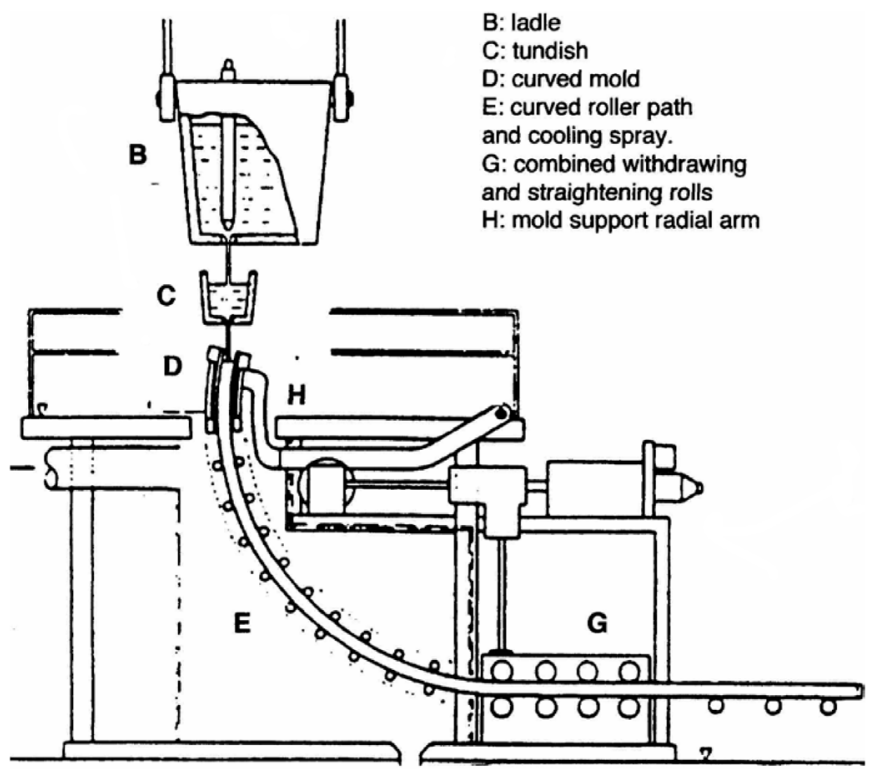

Fig. 9. Machine pilote à lingotière courbe (billettes) chez Von Moos (Lucerne) [2-5].

Fig. 9. Pilot mold curved caster for billets (Von Moos/Dillingen).

Dans le secteur des mini-aciéries électriques le Groupe Korf développe la coulée à grande vitesse sur des machines à billettes avec enfournement chaud directement en sortie des CC par un système entièrement mécanisé (Badische Stahlwerke/Kehl), et alimentation des fours électriques de fusion éventuellement en minerais préréduits (Georgetown Steel/USA ; Hamburger Stahlwerke/Allemagne).

\subsubsection{La maîtrise métallurgique totale (les années 1980 et} 1990)

Les années 1980 voient la reprise d'une réflexion intense sur le profil de la ligne de coulée à propos des machines courbes à brames. Deux pistes seront explorées :

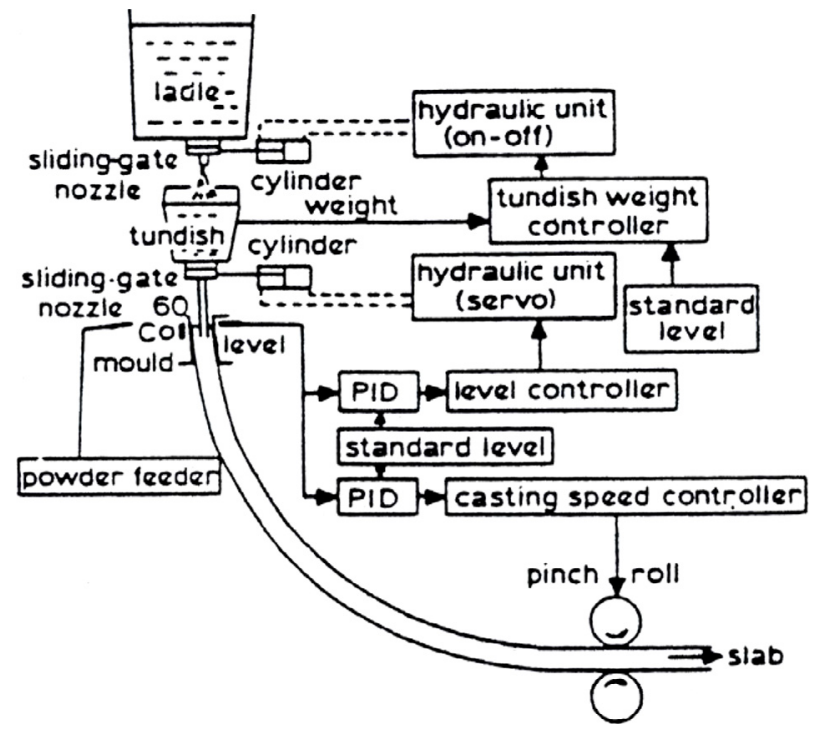

Fig. 10. Système de coulée automatique chez NSC Kashima [2-5].

Fig. 10. Automatic pouring system at Kashima.

- dans la première, on souhaite conserver une lingotière droite pour éviter l'impact des jets d'alimentation en métal liquide sur la peau en formation dans la lingotière, ce qui conduit à ne courber la ligne qu'au-delà d'une partie droite de 3 à $4 \mathrm{~m}$ à partir du ménisque. Pour minimiser les risques d'endommagements du front de solidification liés au cintrage sur cœur liquide on adopte un profil de cintrage très progressif;

- dans la deuxième, afin de poursuivre les gains sur la hauteur des installations, on met au point une machine courbe à tête basse qui associe lingotière courbe à faible rayon et décintrage progressif. Ce seront le cas de la machine dite SHCCM de Sumitomo/Kashima (1979) et de la machine de NSC/Hirohata (partie II, Sect. 2); 
Table 4. Équipement en machines à brames dans la sidérurgie française à partir de 1968.

Table 4. CC slab machines in France, since 1988.

\begin{tabular}{lll}
\hline 1972 & USINOR DK & $\begin{array}{l}\text { CC21 CC22 }+ \text { CC23 } \\
\text { Aciers inoxydables } \\
\text { et à grains orientés }\end{array}$ \\
1972 & CCB Isbergues & CC1 \\
1975 & SOLLAC Fos & CC12 \\
1977 & USINOR DK & CC1 2 \\
1979 & SOLLAC Florange & Aciers inoxydables \\
1983 & Ugine l'Ardoise & CC2 \\
1984 & SOLLAC Fos & CC11 \\
1968 & USINOR DK & \\
\hline
\end{tabular}

- le savoir-faire des constructeurs permet aussi d'envisager la coulée continue pour les produits de forte épaisseur (brames et blooms de plus de $350 \mathrm{~mm}$ d'épaisseur);

La résolution des ultimes problèmes métallurgiques posés par le passage en $\mathrm{CC}$ : maîtrise de la qualité de surface, de la propreté inclusionnaire, des ségrégations en particulier sur les nuances à haut carbone, permettent d'étendre la coulée continue à la quasi-totalité des nuances d'aciers et des formats. Dans cette période sont acquises aussi les performances de productivité qui dépendent entre autres de la vitesse de coulée, de la longueur des séquences, de la disponibilité machine (elle-même dépendante de la conception modulaire des machines et de la parfaite coordination entre les équipes d'exploitation et d'entretien). Le dialogue technique intense entre constructeurs et exploitants contribuera grandement au succès du développement.

Les sidérurgistes des produits plats minces se donnent un nouveau défi: franchir une nouvelle étape dans la simplification des gammes de laminage en supprimant le train continu à bandes (TAB producteur des coils), outil central de toute usine intégrée à produits plats minces. La démarche prendra deux orientations:

- la coulée de brames minces d'épaisseurs $70 / 80 \mathrm{~mm}$, puis $40 / 60 \mathrm{~mm}$ (alors que les brames livrées au TAB étaient dans la gamme d'épaisseur 150/250 mm). Cette épaisseur réduite permet de concevoir un laminage en ligne avec la machine de coulée (un four d'homogénéisation et 4 ou 5 cages de laminage à chaud) ;

- a coulée en bandes de quelques $\mathrm{mm}$ d'épaisseur permettant une mise en coils directement en sortie de la machine de coulée. Ce sera en France, entre bien d'autres équipements pilotes de par le monde, «le projet Myosotis ».

Ces deux techniques seront traitées dans la partie IV [17].

À l'issue de ce rapide survol historique, il convient de rendre hommage à H. Malcor (1906-1998) qui fut à l'origine non seulement des premières rationalisations importantes de la sidérurgie française au sortir de la guerre, mais qui engagea résolument la profession sidérurgique dans la voie de la coulée continue : le démarrage de la coulée continue d'Unieux en 1953, celle d'Allevard en
1955 pour les aciers à ressorts en témoignent ainsi que, sous son impulsion, la création de l'IRSID, préparée par les réflexions de Jean Rist (1900-1944) en matière de recherche métallurgique. Cet institut de recherche professionnel allait jouer un rôle capital dans le développement de la coulée continue en France en apportant un soutien déterminant aux aciéries qui allaient s'équiper. Il convient aussi de souligner le rôle de la CECA finançant des recherches coopératives entre sociétés sidérurgiques européennes.

Le tableau 5 peut donner l'impression au lecteur, par le nombre de sites fermés, d'une régression inéluctable de l'industrie sidérurgique; il faut cependant considérer que nombre de ces fermetures de sites correspondent à des rationalisations inévitables aux bénéfices des sites les mieux équipés ou les mieux situés, ainsi confortés. Entre bien d'autres exemples possibles du dynamisme maintenu de la profession sidérurgique européenne, nous avons retenu le cas, exposé ci-après, de l'aciérie de Kehl de la Badische Stahlwerke (BSW) dont les performances tiennent beaucoup à l'exploitation exemplaire de machines de CC à billettes [12].

Cette société livre des nuances pour ronds à béton (nuances banales s'il en est) sous forme de fil machine à des tréfileries partout en Europe, dont 10 en Allemagne appartiennent au groupe. La BSW est issue de l'éclatement en 1983 du groupe Korf, qui avait bâti un empire en Europe et aux USA sur le concept de mini-usines (four électrique de fusion et $\mathrm{CC}$ de billettes) produisant des aciers courants en produits longs, modèle de production initiée à la fin des années 1960 par les Bresciani. La production de l'aciérie BSW repose sur deux fours électriques à arc et deux coulées continues à billettes (respectivement à 5 et 6 lignes). L'amélioration constante de ces outils a permis en trente ans de porter la production annuelle de l'usine de 850000 à $2320000 \mathrm{t}$ et la production journalière de 3000 à près de 8000 t. Au fil du temps, les améliorations les plus significatives sur la chaîne de production et ses performances ont été les suivantes:

- au niveau des fours électriques de fusion : le poids unitaire des coulées a été porté de 60 à $105 \mathrm{t}$, la puissance apparente des transformateurs de four a été portée de 36 à $90 \mathrm{MVA}$, et tout un environnement technique a été développé autour du four pour optimiser au cours de la fusion l'emploi de l'énergie fossile (charbon mis dans la charge de ferrailles et brûleurs oxy-gaz ou oxy-fuel) avec des injections d'oxygène. Le temps de coulée à coulée a été ainsi ramené à $40 \mathrm{mn}$. Ces fours électriques sont parmi les plus performants au monde;

- au niveau des deux machines de coulée continue (5 lignes et 6 lignes), une grande attention a été apportée à tout ce qui permet de maximiser les temps sous coulée: organisation du plancher de coulée et des manutentions des poches et des répartiteurs; tables d'oscillation des lingotières rétractables de manière à pouvoir changer les lingotières en cours de séquence; amélioration de la tenue des réfractaires... Ces améliorations ont permis d'atteindre un taux de séquence de 115 poches en séquence;

- l'enfournement chaud au laminoir atteint $85 \%$; 
Table 5. Historique de l'équipement en $\mathrm{CC}$ des principaux sites sidérurgiques français. Les dates indiquées correspondent à la mise en service de la première machine sur le site considéré; mais une machine de coulée est un bien durable, dont la durée de vie est de quelques dizaines d'année; il en résulte que la plupart des machines ont subi, au cours de leur vie, des revamping importants s'apparentant parfois à des reconstructions.

Table 5. CC equipment of the main French steel plants.

\begin{tabular}{|c|c|c|c|c|c|}
\hline Année démarrage & Société site & Billettes & Blooms & Brames & Observations \\
\hline 1953 & Jacob Holtzer - Unieux ${ }^{a}$ & $\mathrm{x}$ & & & \\
\hline 1954 & $\mathrm{FCB}^{\mathrm{a}}$ & $\mathrm{x}$ & $\mathrm{x}$ & & \\
\hline 1955 & Allevard $^{\mathrm{a}}$ & $\mathrm{x}$ & & & $\mathrm{CC} 1$ \\
\hline 1960 & SAFE - Hagondange & $\mathrm{x}$ & & & \\
\hline 1963 & Allevard $^{\mathrm{a}}$ & $\mathrm{x}$ & & & $\mathrm{CC} 2$ \\
\hline 1967 & USINOR Dunkerque & & & $\mathrm{x}$ & CC11 \\
\hline 1972 & USINOR Dunkerque & & & $\mathrm{x}$ & $\mathrm{CC} 21-22-23$ \\
\hline 1972 & CCB Isbergues ${ }^{\mathrm{a}}$ & & & $\mathrm{x}$ & Inox et aciers à grains orientés \\
\hline 1973 & Riva Iton/Seine & $\mathrm{x}$ & & & Ronds à béton \\
\hline 1975 & SOLMER & $\mathrm{x}$ & & $\mathrm{x}$ & $\mathrm{CC} 1$ \\
\hline 1975 & SAM - Montereau & $\mathrm{x}$ & & & Ronds à béton \\
\hline 1975 & Vallourec- St Saulve & $\mathrm{x}$ & & & Machine rotative \\
\hline 1975 & ALPA- Porcheville $(\mathrm{CC} 1)^{\mathrm{a}}$ & $\mathrm{x}$ & & & Ronds à béton \\
\hline 1975 & AUMD- Decazeville ${ }^{\mathrm{a}}$ & $\mathrm{x}$ & & & Machine rotative \\
\hline 1977 & SOLLAC Dunkerque & & & $\mathrm{x}$ & $\mathrm{CC} 12$ \\
\hline 1978 & Imphy & $\mathrm{x}$ & & & Machine rotative \\
\hline 1979 & SOLLAC Florange $^{\mathrm{a}}$ & & & $\mathrm{x}$ & $\mathrm{CC} 1^{\mathrm{b}}$ et $\mathrm{CC} 2^{\mathrm{a}}$ \\
\hline 1981 & UNIMETAL- Gandrange $^{\mathrm{a}}$ & $\mathrm{x}$ & $\mathrm{x}$ & & \\
\hline 1982 & SAM Neuves-Maisons ${ }^{\mathrm{a}}$ & $\mathrm{x}$ & & & \\
\hline 1982 & UGINE - Savoie & & $\mathrm{x}$ & & \\
\hline 1984 & UNIMETAL- Gandrange $^{\mathrm{a}}$ & & $\mathrm{x}$ & & \\
\hline 1982 & ASCOMETAL- Les Dunes & & $\mathrm{x}$ & & Machine rotative \\
\hline 1983 & UGINE L'ardoise & & & $\mathrm{x}$ & Aciers inox ${ }^{\mathrm{a}}$ \\
\hline 1986 & SOLLAC Fos & & & & $\mathrm{CC} 2$ \\
\hline 1986 & LME Trith-St-Léger & $\mathrm{x}$ & & & Ronds à béton \\
\hline 1993 & ALPA - Porcheville (CC2) & & $\mathrm{x}$ & & Ronds à béton \\
\hline 1996 & Martial Ucin-Bayonne & & $\mathrm{x}$ & & Ronds à béton \\
\hline 2009 & Vallourec St-Saulve & & $\mathrm{x}$ & & \\
\hline
\end{tabular}

a Machines arrêtées ou démantelées.

b Machine transformée en twin- bloom en 1994.

- il se passe $4 \mathrm{~h}$ entre la reprise de la ferraille sur l'aire de stockage et la mise à disponibilité du fil machine (dans ce laps de temps on aura fondu la ferraille, coulé l'acier, laminé les billettes).

Enfin cette haute efficacité dans l'exploitation des équipements est soutenue par une politique volontariste de formation (en permanence plus de 100 apprentis en formation) et par l'existence de la «Mittelstandliche Kultur » où un groupe familial responsable réinvestit dans l'entreprise, ce qui assure simultanément un taux de fonds propres élevé et les moyens pour la modernisation permanente.

\section{Conclusion}

Sur un demi-siècle, la sidérurgie mondiale s'est équipée pour la quasi-totalité de sa production de machines de coulée continue en adaptant les machines (droites ou courbes) aux formats des produits coulés. Ce développement a été soutenu par des directions d'entreprises sidérurgiques et d'engineering visionnaires et grâce à des avancées métallurgiques simultanées sur la compréhension des phénomènes de solidification. Des gains de productivité considérables en ont résulté. 


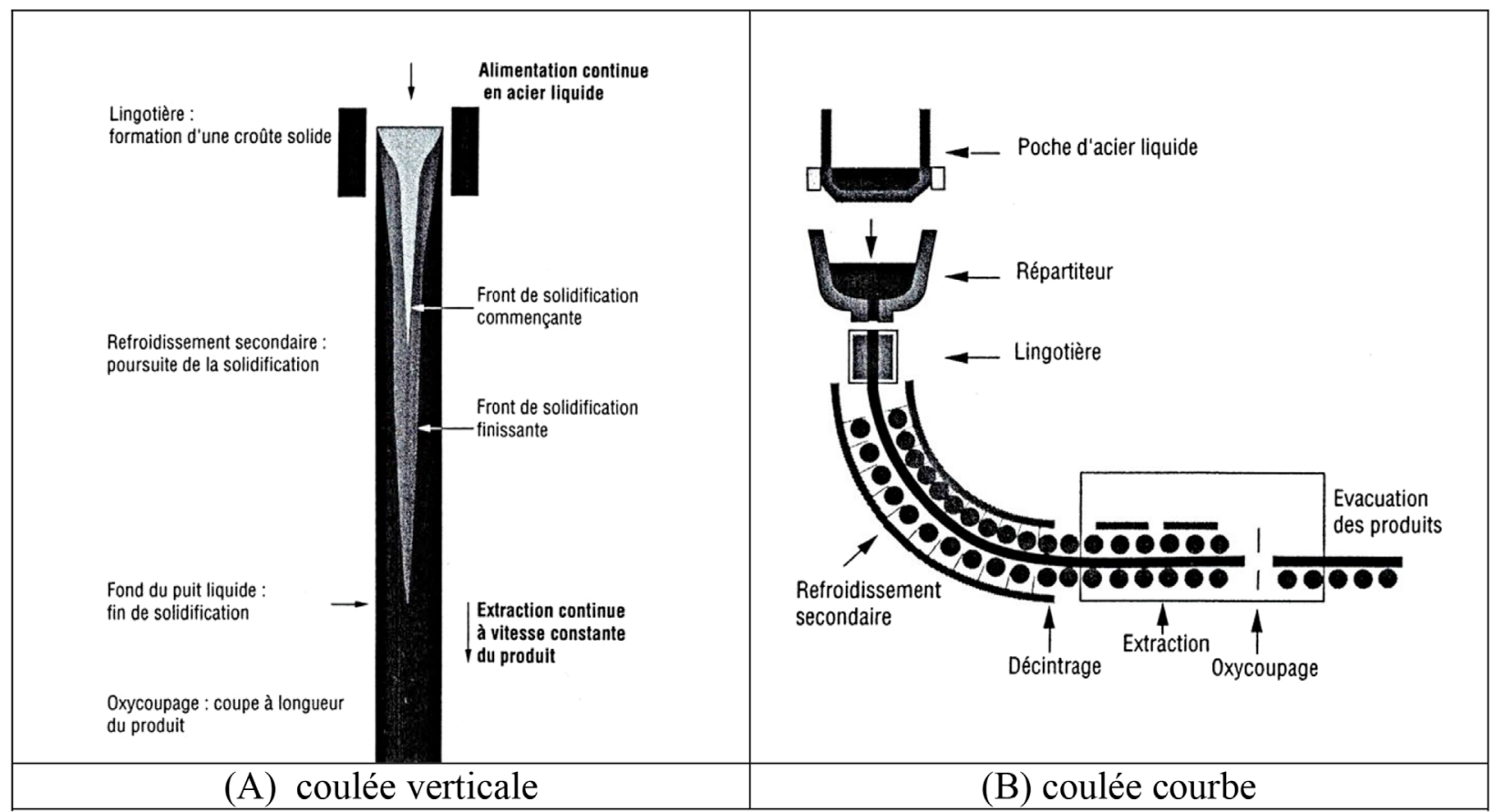

Fig. A1. Schéma de principe d'une coulée continue: (a) machine verticale et (b) machine courbe à brames [13].

Fig. A1. Principles of continuous casting machines (a) vertical, (b) curved for slabs.

\section{Annexe A: Fonctionnement et dimensionnement d'une machine de coulée continue (CC)}

\section{A.1 Principe du fonctionnement d'une machine de CC (Fig. A1)}

La machine comprend plusieurs lignes (de une à six selon les formats et le tonnage coulé) alimentées en acier liquide depuis la poche acier par l'intermédiaire d'un répartiteur (tundish).

En tête de ligne se trouve la lingotière oscillante à parois de cuivre refroidies par circulation d'eau dont la fonction est de former la peau solide du produit. En sortie de lingotière (dont la hauteur est de l'ordre de plusieurs dizaines de $\mathrm{cm}$ ) l'épaisseur de peau est de l'ordre $\mathrm{du} \mathrm{cm}$. Cette peau solide, qui va aller en s'épaississant à mesure que le produit avance en machine, va servir de contenant (à la manière d'un sac) pour le cœur du produit encore liquide.

$\mathrm{Au}$-dessous de la lingotière se trouve le refroidissement secondaire qui occupe la plus grande partie de la longueur de la machine. Il consiste en un système de pulvérisation d'eau dont la fonction est d'extraire l'enthalpie de solidification du cœur du produit. Cette zone comprend des corsets de rouleaux dont les fonctions sont le guidage et le soutien du produit coulé.

Vers la sortie machine, on trouve successivement des cages de rouleaux décintreurs (si machine courbe) afin de sortir un produit rectiligne, des cages extractrices assurant par traction la progression du produit en machine, des équipements de coupe à longueur (cisailles pour les petites sections ou plus souvent chalumeaux) pour débiter le produit en longueurs admissibles par les ateliers aval de laminage.

Le démarrage d'une séquence de coulées de plusieurs poches suppose d'obturer la sortie de lingotière par une pièce métallique sur laquelle viendront s'ancrer par solidification les premières dizaines de $\mathrm{kg}$ d'acier coulé. Cette pièce qui est l'extrémité d'un mannequin (articulé dans le cas d'une machine courbe ou même verticale) sera extraite de la machine par les rouleaux extracteurs en entraînant le véritable produit arrimé par solidification à la tête de mannequin. L'opération d'introduction du mannequin dans la ligne, préalable au démarrage d'une séquence de coulée est désignée par les termes: «réarmement de la machine». Le mannequin fut longtemps introduit par le bas de la machine, ce qui imposait d'attendre la sortie des dernières tonnes coulées avant de réarmer. Les technologues ont mis au point le réarmement par le haut, consistant à introduire le mannequin par la lingotière; on y gagna en productivité.

\section{A.2 Machine droite ou courbe}

Plus la section du produit coulé est forte, plus longue sera la machine et donc plus le constructeur sera confronté à des problèmes de hauteur de machine si l'on souhaite garder une machine droite et verticale (voir ci-dessous). La réponse à ce problème de hauteur excessive est de courber la ligne et d'inscrire une bonne partie de la solidification sur une portion de quart de cercle, y compris éventuellement la lingotière. Nous indiquons dans la partie historique de 


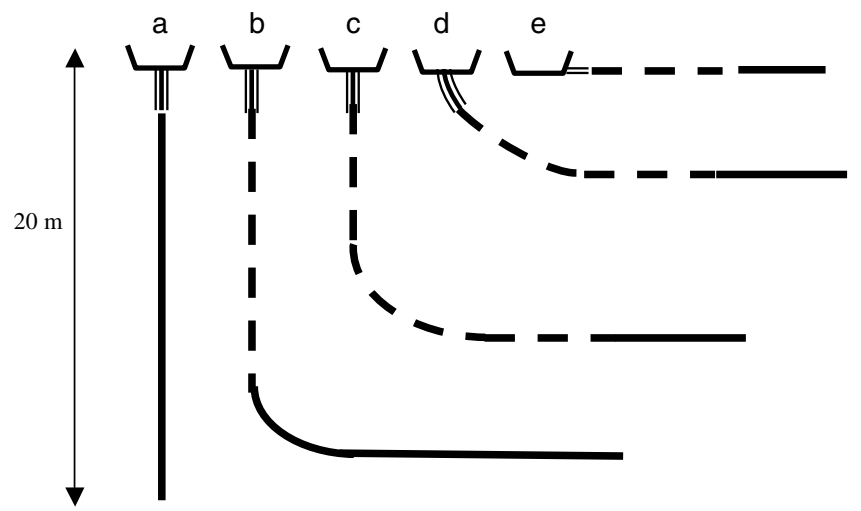

Fig. A2. Schéma des divers types de machines de coulée [14]. Trait continu : produit solide à cœur ; trait pointillé : produit liquide à coeur. a : machine verticale; b : cintrage et décintrage sur coeur solide ; $:$ cintrage et décintrage sur coeur liquide; $\mathrm{d}:$ machine courbe; e : coulée horizontale.

Fig. A2. Various types of continuous casting machines.

l'article que c'est l'invention de ce type de machine qui a permis le considérable développement de la coulée continue pour les produits plats minces (machines à brames). Le fait de solidifier sur un arc de cercle peut avoir des conséquences importantes sur la propreté du métal, dans la mesure où les inclusions non métalliques qui auraient normalement décanté jusqu'au ménisque dans une machine verticale vont se trouver piégées au plafond du produit (intrados) [1]. La réponse à ce problème a été la machine verticale-courbe avec cintrage puis décintrage (développée particulièrement par la société d'engineering Voest-Alpine), dans laquelle le haut de machine (dont la lingotière) reste vertical, et où on procède au cintrage du produit quelques mètres au-dessous de la sortie de la lingotière. Cette machine réalise en quelque sorte la superposition d'une machine droite verticale et d'une machine courbe, au prix d'une double déformation en ligne: le cintrage puis le décintrage. Les progrès réalisés en matière de propreté inclusionnaire de l'acier liquide ont rendu moins pertinente la technologie de la lingotière droite avec cintrage puis décintrage sur la ligne. Il subsiste cependant un problème sérieux résultant de l'emploi de la lingotière courbe : celui de l'impact du jet d'acier sur le front de solidification à l'extrados du produit ; sa résolution a imposé un design spécifique des busettes d'alimentation. La figure A2 schématise les divers types de machines de coulée selon leur géométrie globale.

\section{A.3 Éléments pour un dimensionnement}

Nous nous proposons dans ce paragraphe de définir sommairement une machine (type, format coulé, nombre de lignes) pour alimenter de manière optimale le laminoir aval, avec une productivité convenable et avec un temps de coulée qui soit compatible avec les temps opératoires du four électrique ou du convertisseur amont ( «tap to tap»), et qui ne soit pas excessif pour que la chute de température en poche durant la coulée reste tolérable (nous admettrons qu'un temps de coulé de l'ordre de l'heure est raisonnable et satisfait à la double exigence que nous venons de formuler). Nous prendrons l'exemple suivant: la coulée d'une poche contenant $80 \mathrm{t}$ d'acier liquide dans un format bloom de $240 \times 240 \mathrm{~mm}^{2}$ résultant de l'optimisation des gammes de laminage en aval de la machine.

Il faut qu'en sortie machine, lorsqu'on procède à la découpe du produit, celui-ci soit solide dans toute sa section. Dans l'exemple choisi, la solidification devra donc avoir progressé de $120 \mathrm{~mm}$, depuis la peau jusqu'au cœur (elle progresse sur les quatre faces). La résolution de l'équation aux dérivés partiels gouvernant la propagation des isothermes dans le cas d'un plan infini (équation dite « de la chaleur») aboutit à la formule suivante qui est une approximation largement suffisante pour nos besoins d'ordre de grandeur :

$$
X=K t^{1 / 2}
$$

$X$ épaisseur solidifié est en mm et le temps $t$ en min.

Le coefficient $K$ dépend du matériau coulé et des politiques de refroidissement appliquées. Il est compris entre 20 et 35 pour le refroidissement global sur une machine conventionnelle coulant de l'acier. Dans l'exemple que nous développons ci-après, nous choisissons un refroidissement modéré bien adapté à la nature délicate des produits qui seront coulés: $K=25$. Dans ces conditions le puits liquide sera fermé en un temps de $23 \mathrm{mn}\left(t=120^{2} / 25^{2}\right)$. Si nous envisageons une vitesse de coulée de $0,8 \mathrm{~m} / \mathrm{mn}$ (ce qui est une vitesse convenable pour ce type de format), la longueur du puits liquide sera de $l=0,8 \times 23=18,4 \mathrm{~m}$. Si nous voulons rester en machine droite, nous devrons donc hisser la poche d'acier à plus de $25 \mathrm{~m}$ au-dessus du sol de l'aciérie (si on tient compte des équipements de tête de machine et de découpe en sortie machine). Si nous considérons une telle hauteur comme rédhibitoire, nous serons alors conduits à creuser un gouffre de Padirac dans le plancher d'aciérie, sauf à passer en machine au moins partiellement courbe.

Avec une vitesse de coulée de $0,8 \mathrm{~m} / \mathrm{min}$ le débit par ligne (au format $240 \times 240 \mathrm{~mm}^{2}$ ) est :

$0,8 \times 0,24 \times 0,24 \times 7,3=0,34 t / \mathrm{min}=20,4 t / \mathrm{h}$.

Si nous souhaitons couler dans l'heure la poche contenant $80 \mathrm{t}$ d'acier liquide, la machine devra donc comporter quatre lignes. 
On désigne par longueur métallurgique d'une machine de coulée, la distance, mesurée au long de la ligne (arc de cercle sur une machine courbe), entre le sommet de la lingotière et le niveau de la coupe.

\section{A.4 Considérations générales en matière d'engineering des machines de coulée}

Nous avons justifié ci-dessus, par des considérations sur la solidification, le grand développement linéaire des machines de coulée et partant leur encombrement, et leur complexité ; elles doivent véhiculer des produits dont la densité évolue entre 7,0 et 7,7 (entre liquide et solide) ce qui implique des structures supports d'autant plus lourdes que le format coulé est plus important. Ces structures doivent supporter une ou deux poches d'acier liquide dont le contenu peut atteindre quelques centaines de tonnes. Se posent donc d'évidents problèmes de sécurité (y compris vis-à-vis de la sismicité), de manutention, d'accessibilité. Au fil du temps les engineerings ont dégagé quelques règles fondamentales de conception afin de satisfaire à ces exigences :

Pour les machines les plus lourdes trois structures distinctes portées par trois volumes de béton:

- le premier porte le chariot porte poche ou le pivoteur de poche. Cette structure doit être fortement ancrée sous le sol de l'aciérie ;

- le second ancre au sol les structures propres à la machine de coulée, c'est-à-dire tout ce qui est relatif à la solidification et au supportage du produit;

- le troisième supporte les éléments périphériques et d'habillage, sans liaisons avec les deux structures précédentes.

Avec des machines verticales, on est conduit à installer une partie plus ou moins importante de la machine en fosse et traiter correctement les problèmes d'étanchéité, de rayonnement des produits sur les parois de la fosse, d'accessibilité et de ventilation, d'évacuation des battitures, et d'évacuation des produits coulés avec retour de ceuxci au niveau du sol de l'aciérie.

Nous ne saurions trop insister sur les problèmes de sécurité posés par de telles machines et très sérieusement traités par les constructeurs, puisqu'on y trouve tous les éléments mettant potentiellement en péril, en cas de dysfonctionnement grave, non seulement l'intégrité de l'équipement, mais encore la sécurité des personnels travaillant sur le plancher de coulée, à proximité des lignes pour:

- la manipulation de poches pleines d'acier liquide à grande hauteur;
- les manipulations des répartiteurs sur le plancher de coulée;

- les interventions manuelles au plus près des lingotières lors du démarrage d'une séquence de coulées, au voisinage potentiellement explosif de l'acier liquide et de l'eau de refroidissement (lingotière, refroidissement secondaire).

\section{References}

1. J. Saleil, J. Le Coze, La propreté inclusionnaire des aciers (partie I), Matériaux \& Techniques 103, 506 (2015)

2. M. Wolf, History of continuous casting. 75th Steel making conference, Toronto, proceedings, pp. 83-137 (1992)

3. M.M. Wolf, L'histoire de la coulée continue : Première partie, Rev. Métall. 91, 75 (1994), https://doi.org/10.1051/metal/ 199491010075

4. M.M. Wolf, L'histoire de la coulée continue: Deuxième partie, Rev. Métall. 91, 445 (1994), https://doi.org/10.1051/ metal/199491030445

5. M.M. Wolf, L'histoire de la coulée continue: Troisième partie, Rev. Métall. 91, 589 (1994), https://doi.org/10.1051/ metal/199491040589

6 . Thermophysical properties of materials for nuclear engineering: Tutorial and collection of data, IAEA, Vienne, 2008

7. WSA (World Steel Association), World Steel in figures 2017, https//www.worldsteel.org

8. J. Davené, G. Gay, J. Saleil, Le four électrique à arc-Partie I, Matériaux \& Techniques 101, 205 (2014)

9. J. Davené, G. Gay, J. Saleil, Le four électrique à arc-Partie II, Matériaux \& Techniques 101, 301 (2014)

10. J. Davené, G. Gay, J. Saleil, Le four électrique à arc-Partie III, Matériaux \& Techniques 101, 302 (2014)

11. L. Bäcker, Ph.Gosselin, Quelques aspects de la fabrication des billettes en aciers spéciaux à la coulée continue. Congrès international sur la coulée continue, Biarritz, 1976, p. 180

12. M. Hamy. Des hommes, des outils de production, une volonté industrielle, présentation de Badische Stahlwerke-BSW Kehl, 2017.

13. J.P. Birat, M. Larrecq, La coulée et la solidification; Le livre de l'acier, Tec \& Doc Lavoisier 1994.

14. J. Choné, Coulée continue de l'acier-Aspects métallurgiques, Tech. Ing. 6, M7810 (2004)

15. J. Saleil, J. Le Coze, La coulée continue des aciers-Partie II, Matériaux \& Techniques 106, 504 (2018)

16. J. Saleil, J. Le Coze, La coulée continue des aciers - Partie III, Matériaux \& Techniques 106, 505 (2018)

17. J. Saleil, J. Le Coze, La coulée continue des aciers-Partie IV, Matériaux \& Techniques 106, 506 (2018)

Citation de l'article : Jean Saleil, Jean Le Coze, La coulée continue des aciers. Un exemple de développement technique où l'étroite coopération entre métallurgistes, constructeurs et exploitants ont été d'une grande fécondité, Matériaux \& Techniques 106, 503 (2018) 